\title{
Glottopol
}

Revue de sociolinguistique en ligne

$33 \mid 2020$

Varia - Liberté, égalité, diversité !

\section{La formulation du désaccord dans les débats télévisés algériens : quels « effets-de-politesse »?}

Siham Hocini

\section{OpenEdition}

Journals

Édition électronique

URL : https://journals.openedition.org/glottopol/546

DOI : $10.4000 /$ glottopol.546

ISSN : 1769-7425

Éditeur

Presses universitaires de Rouen et du Havre

Référence électronique

Siham Hocini, « La formulation du désaccord dans les débats télévisés algériens : quels « effets-depolitesse »? », Glottopol [En ligne], 33 | 2020, mis en ligne le 01 janvier 2020, consulté le 02 octobre 2021. URL : http://journals.openedition.org/glottopol/546; DOI : https://doi.org/10.4000/glottopol.546

Glottopol 


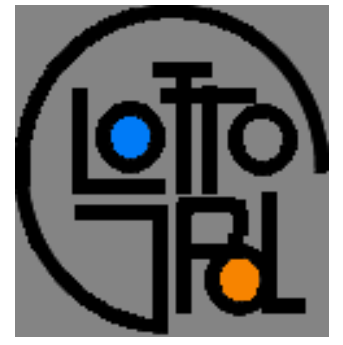

\section{GLOTTOPOL}

Revue de sociolinguistique en ligne $n^{\circ} 33$ - février 2020

Varia : Liberté, égalité, diversité !

\section{SOMMAIRE}

Hommages à Jean-Baptiste Marcellesi : À ringrazià vi, par Niculau Sorba ; “Ciao Ziu ! » par Philippe Blanchet.

Clara Mortamet : Présentation du numéro : Liberté, égalité, diversité !

Manon Him-Aquilli : "Prendre la parole sans prendre le pouvoir ». La mise en registre de l'horizontalité dans les assemblées générales anarchistes/autonomes.

Siham Hocini : La formulation du désaccord dans les débats télévisés algériens : quels "effetsde-politesse "?

Pauline Rannou : Trajectoires de la surdité en France. Parents entendants d'enfants sourds : questionner la fragmentation de l'identité sourde.

Catherine Combaz-Champlaine: Les rectifications orthographiques de 1990 comme révélateurs du rapport des enseignants à l'orthographe.

Marie-Madeleine Bertucci : Éléments d'épistémologie pour l'étude des espaces ségrégués des banlieues urbaines sensibles de la région parisienne.

Élise Gandon: Usages du numérique et illectronisme: nouvelles tâches pour le lecteurscripteur, nouveaux enjeux d'apprentissage.

Marc Arabyan : Quelle langue pour la recherche neurolinguistique française?

José R. de Arellano : Le rapprochement entre l'espagnol et le portugais pendant la décennie dorée sud-américaine : une problématique de l'horizontalité supercentrale.

Adam Wilson: Normes interactionnelles globalisées et communautés de pratique discontinues : les dynamiques sociolinguistiques du tourisme international.

Mélanie Lancien : Caractérisation de la variation liée à la situation de communication : apport de l'acoustique à la phonostylistique.

Assémou Maurice Ludovic Assémou : La prononciation du français parlé en Côte d'Ivoire. 


\title{
LA FORMULATION DU DÉSACCORD DANS LES DÉBATS TÉLÉVISÉS ALGÉRIENS : QUELS « EFFETS-DE-POLITESSE »?
}

\author{
Siham Hocini \\ Université de Tizi-Ouzou et d'Alger 2
}

\section{Introduction}

L'acte de langage de désaccord a fait l'objet de divers travaux de recherche en sciences du langage, sur différents contextes socioculturels. Les uns mettent en exergue ses fonctions (Angouri et Locher, 2012), les autres se basent sur sa dimension séquentielle, avec notamment la notion de "préférence » (Pomerantz, 1978, 1984) ou encore argumentative (Doury, 2009). D'autres recherches, s'inscrivant plus précisément dans le domaine de l'analyse des interactions verbales, l'abordent, au même titre que les autres actes de langage, en tant que noyau de la théorie de la politesse (Brown et Levinson (cités par Kerbrat-Orecchioni, 1992 : 175) ; KerbratOrecchioni, 1992).

Cependant, si les études se consacrant à la description de cet acte de langage tel qu'il se réalise dans le contexte algérien s'avèrent très rares (Hocini et Bedjaoui, 2018a, 2018b), celles qui l'abordent du point de vue de la théorie de la politesse sont inexistantes, ce qui pourrait être expliqué par le fait que le phénomène de la politesse soit très peu étudié dans le contexte algérien. C'est la raison pour laquelle nous nous intéressons dans cet article à la description, sous cet angle, de cet acte discordant dans le contexte algérien. En effet, notre attention se focalise sur les débats télévisés algériens et plus précisément sur les émissions Controverse $e^{1}$ et Place au débat.

La présente étude s'inscrit donc au cœur de la théorie de la politesse linguistique dans laquelle l'acte de désaccord se distingue par son caractère potentiellement menaçant pour la face positive de l'interlocuteur (FTA). Selon Brown et Levinson (cités par Kerbrat-Orecchioni, 1992 : 174), la réalisation directe non accompagnée d'action réparatrice de ce type d'acte de langage constitue une impolitesse. Cependant, Kerbrat-Orecchioni $(2005,2010,2017)$ précise que, si dans certains contextes, l'accomplissement d'un FTA peut engendrer une impolitesse ; ce n'est pas le cas des situations favorisant sa production, à l'exemple d'un ordre (FTA) crié

\footnotetext{
${ }^{1}$ L'utilisation, dans ce travail, de deux termes sémantiquement proches, à savoir « controverse » et « désaccord », peut prêter à confusion. Ainsi, afin d'éviter au lecteur d'être confronté à toute forme d'ambiguiité, nous estimons nécessaire d'expliquer le sens que véhicule chacun d'eux dans le présent article. En effet, le mot « désaccord» renvoie à l'acte de langage par lequel un locuteur contredit son interlocuteur : cet acte contradictoire constitue l'objet de cette recherche. "Controverse », quant à lui, est un terme qui signifie un débat, une discussion entre des personnes qui ne partagent pas les mêmes idées, les mêmes opinions sur une question donnée : il constitue le titre de l'une des deux émissions qui composent le corpus sur lequel repose cette recherche.
} 
lors d'un entrainement militaire. C'est ce que confirment Moïse et Oprea (2015: §6) en soulignant qu' " une même production langagière peut être interprétée comme polie, impolie voire violente selon le contexte dans lequel elle apparait ». Qu'en est-il de l'effet de politesse que pourrait provoquer l'acte de désaccord dans les émissions télévisées algériennes Controverse et Place au débat?

Le débat télévisé est un type d'interaction verbale orienté vers le désaccord malgré la menace qu'il constitue pour la face des participants. En effet, pour Kerbrat-Orecchioni (2017:218), le désaccord est la condition nécessaire pour que tout débat télévisé puisse prospérer : il constitue « la loi du genre » note-elle. Cet acte contradictoire est donc attendu dans ce contexte : « ils (les désaccords) constituent la règle plutôt que l'exception, étant attendus par les animateurs et le public, mais aussi par les débatteurs eux-mêmes, malgré le péril qu'ils constituent pour leur face - ils s'y sont préparés, ont fourbi leurs armes » (Doury et Kerbrat-Orecchioni, 2011 : 72). D'ailleurs, les débatteurs sont invités pour discuter : ils ont donc le droit de manifester leur opposition face à leurs adversaires. Mais qu'en est-il de la place qu'il occupe dans les débats qui constituent notre corpus ? Cette question s'impose dans ce contexte, d'autant plus que la valeur pragmatique d'un acte de langage varie d'une communauté socioculturelle à l'autre et d'une situation à l'autre (Kerbrat-Orecchioni, 1992). C'est l'identification de la place du désaccord dans ces débats qui nous permettra de décrire l'effet de politesse qu'il provoquerait dans ce contexte.

Par ailleurs, quel que soit son contexte de production, l'expression du désaccord s'accompagne souvent, à l'instar des autres actes de langage, de procédés qui tantôt atténuent sa menace (les adoucisseurs), tantôt l'aggravent (les renforçateurs ou durcisseurs) (KerbratOrecchioni, 1992, 2005). Ce qui a un impact sur l'effet de politesse qu'elle pourrait produire dans ce contexte, puisque comme le précise Kerbrat-Orecchioni (2010:39), «pour identifier un énoncé comme poli ou impoli, il faut tenir compte à la fois de son contenu (en tant que FTA, $\mathrm{FFA}^{2}$ ou mélange des deux), de sa formulation (adoucie ou renforcée), et de son contexte d'actualisation ». À partir de ces critères, Kerbrat-Orecchioni (ibid.) distingue différentes formes de politesse qu'elle baptise «politesse », « impolitesse », « non-politesse », « hyperpolitesse » et « polirudesse ».

Par conséquent, pour répondre à la question principale qui motive cette recherche, et qui consiste à rendre compte des effets de politesse que peut provoquer un FTA bien précis, l'acte de désaccord, dans un contexte bien précis, les émissions télévisées algériennes Controverse et Place au débat, nous identifierons d'abord sa place dans le corpus (attendu ou non); ensuite, nous classerons ses différents effets en prenant en considération sa formulation et en nous appuyant sur la distinction de Kerbrat-Orecchioni (2010) des différentes formes de politesse. Il est donc question dans ce travail de mener une analyse qualitative qui repose sur les travaux de Kerbrat-Orecchioni sur la politesse linguistique. Ce choix est motivé par le fait que ces travaux constituent le cadre théorique le plus riche et le plus structuré dont s'inspirent beaucoup de recherches en la matière.

\section{L'acte de langage du désaccord : un acte menaçant pour la face}

L' « acte de langage » est l'un des concepts fondamentaux de la pragmatique, un domaine qui découle des théories logiques et philosophiques anglo-saxonnes. Il renvoie à l'idée selon laquelle le but du langage dépasse la représentation et la description du monde ainsi que la transmission des informations entre les interlocuteurs (Austin, 1970). Il est question d'agir sur

\footnotetext{
2 «Face Flattening Act », en français « Acte flattant pour la face » : une notion mise en place par Kerbrat-Orecchioni (1992).
} 
autrui et sur le monde, de réaliser des actes par des moyens langagiers : « Parler c'est accomplir des actes » (Searle, $1972: 59$ ).

Le désaccord est considéré comme un acte de langage puisqu'il peut agir sur les interlocuteurs, en entrainant un changement de leur comportement et de leurs relations personnelles; ainsi que sur le déroulement de l'interaction, en engendrant un refroidissement de l'ambiance. Autrement dit, l'acte de désaccord peut réaliser un acte perlocutoire. Par ailleurs, Kerbrat-Orecchioni (2016) le définit comme un acte de nature réactive à l'assertion avec laquelle il compose l'unité dialogale minimale d'une interaction, à savoir l'échange.

Le désaccord, au même titre que le reproche, la critique, le refus, etc., peut constituer une menace pour la face de l'interlocuteur : le vexer, le toucher dans son amour-propre. C'est à partir de cette idée, et en empruntant à Goffman $(1973,1974)$ les notions de «face », de « face want » et de « face work », que Brown et Levinson (cités par Kerbrat-Orecchioni, 1992 : 167) proposent la notion de «FTA»: «Face Threatening Acts », en français «Actes menaçants pour la face $»$. Le caractère potentiellement menaçant du désaccord est également mis en lumière par Vion (2008: 224) qui affirme que l'expression de la «divergence fonctionne comme une menace pour la face des autres », ainsi que par Leech (cité par Kerbrat-Orecchioni, 1992 : 181) qui met en place une règle de politesse qui consiste à minimiser le désaccord entre les interlocuteurs.

\section{Cadre méthodologique de la recherche}

Controverse et Place au débat sont deux émissions télévisées algériennes hebdomadaires. La première est diffusée à vingt heures trente sur la chaine Dzaïr $\mathrm{TV}^{3}$, tandis que la seconde est diffusée à dix-neuf heures trente sur la chaine Echorouk News $\mathrm{TV}^{4}$. Ces émissions se caractérisent par leur homogénéité au niveau du type d'interaction (interactions verbales médiatiques), de la nature des thèmes qu'elles traitent (politique, économique et social) ainsi que de leur contexte de production (elles ont en commun le site ainsi que les caractéristiques individuelle, socioprofessionnelle et culturelle des participants ; voir annexes). Aussi, elles sont produites essentiellement dans la même langue, le français ${ }^{5}$, et animées par le même journaliste, Khaled Drareni. En somme, à première vue, le téléspectateur pourrait confondre les deux émissions.

Leur choix n'est pas motivé par un objectif de comparaison, mais par le souci d'avoir un nombre suffisant de débats pertinents pour notre analyse. En effet, toutes les émissions ne s'organisent pas sous forme de débat. Par ailleurs, ces émissions sont accessibles au chercheur, du point de vue déontologique et juridique, étant donné qu'elles sont largement diffusées.

Notre choix porte exactement sur trois débats de Controverse traitant les thèmes de « Gaz de schiste », diffusé le 27/11/13; de "L'autonomie de la Kabylie », diffusé le 05/12/13 et du " Mouvement Barakat ${ }^{6}$ ", diffusé le 12/03/14; ainsi que sur deux débats de Place au débat s'articulant autour de la « Situation des droits de l'Homme en Algérie », diffusé le 30/12/16 et

\footnotetext{
${ }^{3}$ Dzaïr TV est une chaine de télévision généraliste privée algérienne basée à Alger créée le 8 mai 2013.

${ }^{4}$ Echorouk TV est une chaine de télévision généraliste privée algérienne basée à Alger lancée le 6 mars 2011.

${ }^{5}$ Avec un recours rarissime aux langues maternelles des participants (l'arabe algérien et le kabyle) ainsi qu'à l'arabe classique. Par ailleurs, en parcourant le corpus, nous avons constaté que les participants expriment parfois leurs désaccords dans leurs langues maternelles. Si nous admettons avec Zongo (2004) qu'un locuteur ne peut être plus expressif que dans sa langue maternelle, nous pourrions considérer ce recours à ces langues comme révélateur d'un besoin de renforcement de l'expression du désaccord. Les locuteurs expriment mieux leur opinion contradictoire en se tournant vers leurs langues maternelles, et lui donnent ainsi plus de poids et de force. Cela augmente le degré de sa menace et lui donne une allure durcie.

${ }^{6}$ Barakat est un mouvement algérien né au début de l'année 2014, pour dénoncer la candidature de Bouteflika pour un quatrième mandat.
} 
du «Jil FCE », diffusé le 27/01/17. Ces débats réunissent trois à cinq participants débattant autour d'une table : un journaliste-animateur et des invités-débatteurs.

Ces débats répondent aux besoins méthodologique et linguistique de cette recherche, ce qui justifie d'ailleurs leur choix. En effet, ils se présentent comme un lieu propice pour la production de l'acte de langage du désaccord visé par cette étude, du moment qu'ils ont trait à des questions cruciales et critiques du pays. De plus, ils sont produits en français : étant donné que notre travail s'inscrit dans le domaine des études sur la langue française, il a été primordial de choisir des débats produits en français ou du moins en alternance codique incluant cette langue. Ce qui nous permettrait de rendre compte de la réalisation de l'acte du désaccord dans cette langue telle qu'elle est utilisée dans le contexte algérien.

Nous avons téléchargé ces épisodes, disponibles en ligne sur le site « You Tube », en janvier 2018, en recourant à l'extension « Grab any media » du navigateur « Opéra »; et nous les avons transcrits en nous basant sur la convention de transcription ICOR ${ }^{7}$.

Avant de décrire les effets de politesse engendrés par l'expression du désaccord dans ces numéros, il est primordial de montrer la place que cet acte y occupe.

\section{La place du désaccord dans le corpus}

L'analyse du corpus montre qu'un bon nombre d'expressions du désaccord sont adoucies. Cela confirme le caractère menaçant de cet acte dans l'environnement culturel des participants : "la présence d'un adoucisseur confirme en même temps l'existence d'un FTA », pour reprendre Kerbrat-Orecchioni (2010 : 44). Par exemple, si un participant s'excuse en formulant son désaccord (or l'excuse est un adoucisseur), cela signifie qu'il sent l'offense que comporte cet acte dans sa culture, et la reconnait indirectement : « qui s'excuse s'accuse », note KerbratOrecchioni (1994: 156).

Exemple 01 : Controverse, 05 décembre 2013

252 AG [...] tamazight en tant que- en tant que langue nationale/ je ne l'ai jamais revendiquée parce que (.) le tamazight est une langue nationale de fait de fait [ma mère

$253 \mathrm{KD}$ [langue ancestrale/

254 AG ma mère parle chaoui/ mon père parle chaoui/ ma famille parle chaoui/ nous sommes des amazighs/ (.) de- de- de- de- et:: de fait/ (.) de fait (.) le tamazight est pratiqué/ (.) donc c'est une langue nationale (.) c'est comme l'arabe algérien (.) qui est une langue nationale de fait (.) maintenant reste l'acte (.) politique/ (.) l'acte politique/ c'est son officialisation (.) [...]

259 ML $[\ldots]$ encore une fois (.) moi je reviens- j'aime bien revenir aux concepts/

$260 \mathrm{KD}$ allez-y/

261 ML euh le statut de langue nationale/ euh nous l'avons depuis que- qu'elle est [institutionnalisée

262 AG [depuis la nuit des temps/

$263 \mathrm{ML}$ non/ depuis que c'est constitutionnellement/ admis (.) c'est- on- on était dans un vernaculaire/ (.) pour qu'une langue soit nationale vérita- véritablement/ il faut- il faut que ça soit reconnu par les institutions du pays/ (.) il n'y a pas de langue nationale de fait/ (.) $<$ ((en s'adressant à $\mathrm{AG}$, avec un sourire amical et un ton doux) je suis désolé de (.) te contredire>

\footnotetext{
${ }^{7}$ Du laboratoire ICAR (UMR 5191, CNRS- Université Lyon 2-ENS-LSH de Lyon).
} 
Dans les tours de parole 252, 254 et 262, AG affirme que le tamazight est naturellement une langue nationale puisque c'est la langue des ancêtres « depuis la nuit des temps » : elle n'a pas besoin qu'elle soit constitutionnellement admise pour avoir le statut de langue nationale : « le tamazight est une langue nationale de fait ». C'est cette opinion que conteste ML dans les tours 261 et 263 en utilisant la négation polémique : «il n'y a pas de langue nationale de fait », « on était dans un vernaculaire, pour qu'une langue soit nationale véritablement, il faut que ça soit reconnu par les institutions ». Nous constatons que ce désaccord est suivi de la formule d'excuse « je suis désolé (de te contredire) ${ }^{8}$, produite sur un ton aimable et accompagnée d'un sourire amical. ML reconnait ici la menace que constitue cet acte « de te contredire » et tente de rectifier le tir et de se racheter.

Par ailleurs, l'analyse de ces débats montre que tout en constituant intrinsèquement une menace pour la face des débatteurs, la production de cet acte contradictoire est perçue comme normale et attendue dans ce contexte conflictuel. C'est ce que montrent les exemples cités cidessous.

Exemple 02 : Place au débat, 30 décembre 2016

168 AG ça veut dire/ ça veut dire madame/ j'ai le droit d'être candidat/j'ai le droit de gérer mon village (.) j'ai le droit de gérer mon a p c (.) écoutez madame/ quand le wali est le maitre à bord dans une wilaya/ (.) si vous parlez des droits de l'homme et de liberté (.) allons madame et les ELUS n'ont pas le même pouvoir $\backslash$ est-ce que c'est [normal/

$169 \mathrm{KD}$

170 SB

$171 \mathrm{KD} \&$ terrain/

172 SB \&\&non pardon- pardon- pardon/

$173 \mathrm{KD}$ oui/

174 SB moi je ne suis pas venue pour assister à un tel débat/ (.) je suis désolée/

$175 \mathrm{KD}$ mais c'est un débat/ on peut [parler/

$176 \mathrm{SB} \quad$ [non non smahli ${ }^{9}$ je ne suis pas venue pour ca/ (.) je suis venue (.) je pensais qu'on allait parler de- de- de- de manière plus responsable/ et plus [objective $\underline{\text { mais dresser-\& }}$

$177 \mathrm{KD}$

son point de vue $/ \& \&$

178 SB \&dresser un tableau noir (.) dre- dresser un\&

$179 \mathrm{KD}$ \&\&chacun a son point de vue madame SB/

$180 \mathrm{SB} \&$ tableau noir (.) c'est faux/ (.) on ne peut pas dire que tout va mal/

Dans ce passage, SB manifeste un désaccord portant sur l'opinion de son adversaire AG qui asserte en 168 que l'Algérie ne connait pas de progression sur la question du respect des droits de l'Homme. Outre l'expression de cet acte contradictoire, SB manifeste, en 174 et 176, son mécontentement de la tournure conflictuelle que prend le débat, et que provoquent les désaccords produits par ses adversaires à son encontre « je ne suis pas venue pour assister à un tel débat ». Cela montre que SB est sensible à cet acte contradictoire, ce qui prouve qu'elle le sent comme une offense, une menace pour sa face positive. Dans les tours 175, 177 et 179,

\footnotetext{
${ }^{8}$ L'acte d'excuse se réalise dans ce cas par la description d'un état d'âme approprié que Kerbrat Orecchioni (1994 : 164) range dans la rubrique des réalisations implicites de l'excuse.

${ }^{9}$ Excusez-moi !
} 
l'animateur KD s'oppose à SB en soulignant le caractère normal du désaccord dans un débat " mais c'est un débat, on peut parler, chacun a son point de vue madame SB ». Cela montre que la production de cet acte dans ce contexte est normale et attendue même si elle se sent comme une offense : l'interactant est touché dans son amour-propre, mais il doit prendre sur lui car c'est le contexte qui l'exige ${ }^{10}$.

En outre, ce caractère attendu du désaccord se confirme par le comportement de l'animateur qui veille à son déclenchement afin de mener à bien ses débats (Hocini et Bediaoui, 2018b : 1014). De plus, l'animateur ne se contente pas de déclencher des désaccords entre ses invités mais il met également en scène ces divergences par l'explicitation même de leur existence dans le débat. C'est ce qu'illustre l'exemple ci-dessous, extrait de l'émission Controverse sur le gaz de schiste, où l'animateur KD essaye de déclencher une opinion contradictoire chez KB tout en mettant en exergue le désaccord déjà existant entre les autres interlocuteurs : entre AA et CC en 030 ; et entre AA d'une part, SR et CC d'une autre part en 114 :

Exemple 03 : Controverse, 27 novembre 2013

$030 \mathrm{KD} \mathrm{KB} /$ (.) le gaz de schiste/ (.) danger ou pas/ (.) monsieur AA dit non (.) monsieur CC dit oui (.)

$114 \mathrm{KD}[\ldots] \mathrm{KB} /$ on aimerait d'abord (.) connaitre votre position/ on sait que monsieur AA est pour (.) l'exploitation du gaz de schiste/ (.) docteur SR/professeur CC/ (.) plutôt contre/ (.) et vous/

Si l'expression du désaccord est attendue dans cette situation, qu'en est-il de son adoucissement et durcissement de ses manifestations en interaction ?

\section{La place de l'adoucissement et du renforcement du désaccord dans le corpus}

L'analyse du corpus montre que l'absence d'adoucisseurs est attendue (dans une autre situation, une demande (FTA) non adoucie destinée à une personne que l'on connait peu (une vendeuse, par exemple) serait considérée comme anormale ; ex : «Je veux un stylo !»). Par ailleurs, dans le cas inverse, la présence des adoucisseurs dans ces débats télévisés n'est pas considérée comme excessive et exagérée. En effet, nous avons vu supra, que malgré sa nécessité, l'acte de désaccord conserve son effet menaçant; sans oublier la présence des téléspectateurs, même virtuelle, qu'il ne convient pas de choquer, et envers lesquels les débatteurs doivent faire preuve de respect et de politesse ${ }^{11}$. Les adoucisseurs de l'expression discordante sont appropriés à cette situation, même si leur absence y est acceptée.

Quant à la production des renforçateurs, elle n'aura pas l'effet qu'aurait provoqué une demande renforcée, même entre personnes proches ${ }^{12}$ (ex : «donne moi ton stylo tout de suite »). Pour Kerbrat-Orecchioni (2010:45), dans le débat télévisé, qu'elle assimile à une sorte de « guerre verbale », « il s'agit avant tout d'attaquer l'adversaire pour en triompher ». Par ailleurs, l'auteur considère, à la suite de Lacroix, que la norme dans ces débats « est que les adversaires se malmènent, se chahutent, se provoquent et se rendent coup pour coup » (Kerbrat-

\footnotetext{
${ }^{10}$ Cela nous fait penser à la place de l'acte de la critique dans les soutenances des travaux de recherches universitaires : tout en étant un acte offensant pour la face de l'étudiant, sa production est nécessaire, donc normale et attendue dans ce contexte. Ce point peut constituer une piste pour une recherche ultérieure.

${ }^{11}$ Cela révèle l'importance du rôle du public dans ce type d'interactions par rapport à l'image de soi.

${ }^{12}$ Sauf dans certaines relations hiérarchiques (l'axe vertical d'une relation) mais intimes (son axe horizontal) : lorsqu'elle est utilisée par exemple par des parents en s'adressant à leurs enfants (Kerbrat-Orecchioni, 1992).
} 
Orecchioni, 2017 : 215). Ainsi, les renforçateurs de l'expression contradictoire dans ce contexte, ne sortent pas des normes de cette "guerre métaphorique », ils sont donc appropriés à ce type d'interaction verbale.

D'ailleurs, ce qui est remarquable dans l'exemple 04 commenté ci-dessus, est que le désaccord de HS produit en 048 n'est pas adouci, au contraire, il est renforcé puisque HS l'accompagne d'une évaluation négative du raisonnement de MR, qu'il réalise par l'adjectif péjoratif « insultant ». Cependant, cela ne choque pas MR qui, au contraire, justifie ses propos.

Exemple 01 : Place au débat, 27 janvier 2017

048 HS dire que c'est des bébés/ c'est insultant vis-à-vis des [adhérents je me permets je comprends

$049 \mathrm{MR}$

guillemets $=$

[entre guillemets/ entre

Nous constatons donc que ni l'absence des adoucisseurs de l'expression du désaccord, ni la production de ses renforçateurs, ne constituent une anomalie dans ce contexte.

La place du désaccord dans les émissions Controverse et Place au débat, de ses adoucisseurs et durcisseurs est mise au clair ; nous rendons compte à présent des différents effets de politesse que cet acte peut produire dans ce contexte.

\section{Les effets de politesse de l'énoncé contradictoire dans le corpus}

À la lumière de ce qui a été dit supra, nous nous sommes rendue compte que l'énoncé véhiculant l'acte de désaccord peut produire différents effets de politesse dans les émissions Controverse et Place au débat. Nous avons distingué des désaccords polis mais renforcés, nonpolis ainsi que ceux qui relèvent de la polirudesse, que nous proposons d'appeler dans ce travail les désaccords « poli-rudes ».

\subsection{Le désaccord non-poli}

À la suite de Lakoff, Kerbrat-Orecchioni (2005, 2010, 2017) définit la non-politesse, ou l'apolitesse, comme la présence attendue, d'un FTA non adouci ou même durci, dans un contexte donné. Nous avons vu supra que la présence du désaccord non adouci est normale et attendue dans les émissions qui composent notre corpus. Ainsi, nous pouvons conclure que les désaccords non adoucis ou renforcés relèvent de la non-politesse. Cela correspond à ce que Kerbrat-Orecchioni (2010: 38-39) confirme à propos de la production des FTA dans les débats : " les débatteurs se reconnaissent mutuellement le droit de formuler des FTA sans les adoucir, FTA qui relèvent donc pour la plupart de la non-politesse sans verser dans l'impolitesse »; et, d'une manière générale, dans les interactions à caractère conflictuel où « la brutalité des comportements n'implique pas forcément leur "impolitesse" ».

Exemple 01 : Place au débat, 27 janvier 2017

184 HS vous parlez\de d'avantages fiscaux qui ont été accordés par l'état/ et (.) qui ont été portés par le forum des chefs d'entreprises/ l'état n'a pas attendu le forum des chefs d'entreprises $\backslash$ pour donner $\backslash$ euh le- les fameuses procédures [et

$185 \mathrm{MR}$

[je n'ai pas dit ça/ je n'ai pas dit ça

Dans cet exemple, MR exprime en 185 un désaccord portant sur la reformulation de ses propos par son partenaire du débat HS : «je n'ai pas dit ça ». Elle produit un désaccord qui n'est pas uniquement adouci, mais renforcé par l'interruption et la réitération. Ce désaccord ne 
relève pas de l'impolitesse, puisque sa production est normale dans ce contexte, mais ne relève pas non plus de la politesse : il s'agit donc d'un désaccord non-poli.

Par ailleurs, ce qui est observable dans les émissions concernées par cette étude, c'est que les renforçateurs se trouvent parfois combinés avec les adoucisseurs dans la même expression discordante, comme le montre l'exemple suivant :

Exemple 02 : Place au débat, 30 décembre 2016

$146 \mathrm{SB} \leq(($ en regardant son adversaire) ) euh::: euh- euh avec tout le respect que je vous dois $\backslash$ et je respecte votre point de vue (.) mais je ne le partage pas du tout (.)

Dans ce passage tiré de l'émission Place au débat sur les droits de l'Homme en Algérie, SB accompagne son expression de désaccord «je ne le (votre point de vue) partage pas » d'un ensemble d'adoucisseurs, la marque d'hésitation «euh» et les formules marquant le respect " avec tout le respect que je vous dois, et je respecte votre point de vue », auxquels elle joint un durcisseur «pas du tout ».

La juxtaposition, dans une seule expression discordante, des deux catégories d'adoucisseurs et de durcisseurs, donne naissance à une forme hybride de désaccord générant un effet de politesse différent de celui qui est décrit précédemment.

\subsection{Le désaccord poli mais renforcé}

La politesse, selon Kerbrat-Orecchioni (2005 : 209), consiste à produire un FFA attendu (politesse positive) ou à éviter ou adoucir un FTA (politesse négative). À ce titre, les divers adoucisseurs du désaccord (FTA) figurant dans le corpus génèrent un effet de politesse négative : ils ne relèvent pas de l'hyperpolitesse puisque nous avons vu supra qu'ils sont appropriés au contexte du débat. Ainsi en adoucissant leurs désaccords, les participants construisent un ethos poli devant le public (Kerbrat-Orecchioni, 2016: §89) : en atténuant l'offense que constituent leurs désaccords pour la face de l'autre, ils témoignent d'un certain souci de politesse négative.

Cependant, ce qui a attiré notre attention, c'est que tous les désaccords polis identifiés dans le corpus sont renforcés. Cela revient au fait que l'expression du désaccord combine parfois adoucisseurs et durcisseurs. De part le caractère attendu de ces renforçateurs, ce cas de désaccord n'est pas une impolitesse ; mais il ne relève pas non plus de la non-politesse du fait de la présence des adoucisseurs (nous avons vu que la non-politesse est l'absence normale d'adoucisseurs). C'est pourquoi nous parlons dans ce cas d'un désaccord " poli mais renforcé ».

Exemple 01 : Controverse, 12 mars 2014

$168 \mathrm{OA}$ d'accord/ (.) et là ce que- ce qu'a raconté la presse est faux/ [...] j'ai acheté el watan/ (.) eh ben je regrette/ (.) c'est (.) tout à fait faux ce qu'il a raconté (.) amplifié de tous les côtés/ [...] on a amplifié la chose/ (.) on a joué sur la peur (.)

$188 \mathrm{KD}[\ldots]$ est-ce que le mouvement est amplifié selon vous SK/

189 SK [pas du tout/ premièrement- parce que (.) laissez\&

190 OA [à part/ à- à part une cinquantaine de personnes\&\&

191 SK \&moi parler monsieur/ avec tout mon respect pour\&

192 OA \&\&on parle d'une grande manif à (inaud.)/ non/

193 SK \& vous et pour tous les algériens d'ailleurs/ (.) euh:: on est là/ (.) on s'est fait embarquer/ alors si vous n'avez pas vu ça/ et que vous avez vu juste la une d'el watan/ (.) c'est grave/ (.)

Dans le tour de parole 168, OA asserte que, en parlant des manifestations menées par les membres du mouvement Barakat, la presse, entre autres le quotidien El Watan, «a amplifié la 
chose » "de tous les côtés ». C'est cette affirmation que conteste SK, le représentant de ce mouvement, en 189, 191 et 193, en utilisant une variante renforcée de la négation polémique " pas du tout ». Outre ce modalisateur de certitude, le participant recourt, pour renforcer son désaccord, à la critique (FTA) réalisée par le prédicat adjectival péjoratif «c'est grave ». Cependant, ce désaccord ne relève pas de l'impolitesse du moment que ces attaques sont attendues dans ce contexte conflictuel. Au contraire, l'utilisation de la formule exprimant le respect, "avec tout mon respect pour vous », lui confère un certain effet de politesse, et ce, selon la conception aussi bien ordinaire que formelle du terme ${ }^{13}$. Il s'agit donc ici d'un cas de désaccord poli mais renforcé.

Une autre forme de désaccord, qui n'est ni poli, ni impoli, ni même non-poli, est observée dans les émissions Controverse et Place au débat. Il s'agit d'une expression contradictoire qui relève de ce que Kerbrat-Orecchioni (2010) appelle la polirudesse.

\subsection{Le désaccord poli-rude}

Pour Kerbrat-Orecchioni (2010 : 46), la polirudesse consiste à dissimuler un FTA sous des " énoncés qui en apparence sont des FFA »; autrement dit, il s'agit de FTA qui prennent la forme de pseudo-FFA. C'est ce que Moïse et Oprea (2015: $§ 5)$ appellent « la violence verbale détournée »: «Cette forme de politesse surgit quand les marques habituelles de la politesse sont fausses ». En effet, pour ces auteurs, "des paroles polies peuvent cacher en réalité des attaques » (Moïse et Oprea, $2015: \S 1$ ). Le désaccord dans les émissions étudiées dans ce travail, prend parfois la forme d'un pseudo-FFA, à l'exemple d'une pseudo-excuse «je suis désolé », réalisée sur un ton sec; ou d'un compliment perfide «bravo ! » dénotant l'ironie : réalisés de la sorte, ces actes de langage s'apparentent "au phénomène de la polirudesse ». Nous proposons d'appeler dans ce travail, l'acte contradictoire produisant cet effet de polirudesse, un désaccord «poli-rude ».

Exemple 01 : Place au débat, 27 janvier 2017

203 MR quand je vois les chiffres/ (.) quand je vois les chiffres (.) je v- bon (.) en- avec c- endans cette situation de crise/ (.) avec la baisse de la dépense publique/ il est tout à fait normal que le secteur (.) .h il- qu'on enregistre une décélération au niveau du secteur du btph tout à [fait normal

$204 \mathrm{KD}$

[Voilà

mhh

205 HS

un ton moqueur)) bravo/

$[<($ avec

HS réalise en 205 un trope illocutoire où la valeur de désaccord se trouve dissimulée par celle d'un compliment à valeur d'ironie «Bravo!». Ainsi, sur ce trope illocutoire vient se greffer la polirudesse : il s'agit bien ici d'un désaccord poli-rude.

Par ailleurs, ces effets de politesse sont générés dans le corpus avec des doses variables. En effet, quand certains effets y dominent, d'autres n'occupent que des places marginales ; comme le montre le graphique suivant :

\footnotetext{
${ }^{13}$ Pour la distinction des deux formes de la politesse, voir Kerbrat-Orecchioni (2010:36).
} 


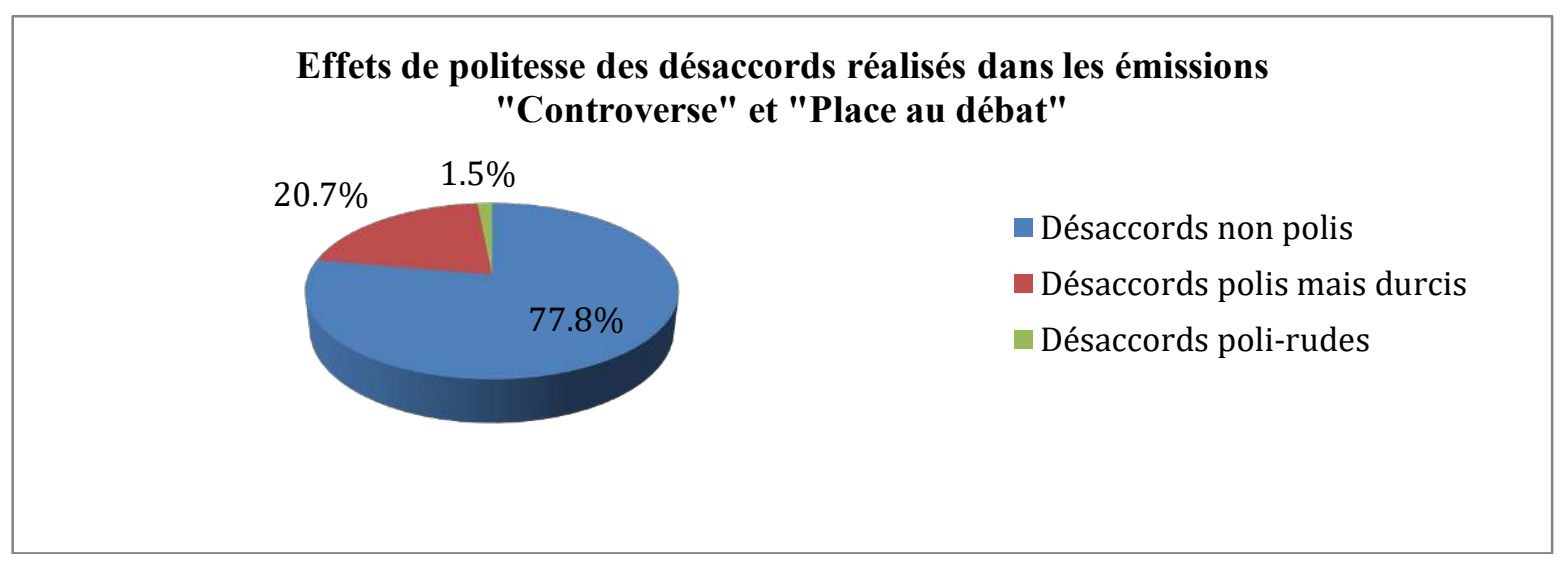

Figure $n^{\circ} 1$

Ce graphique met en exergue la diversité des effets de politesse générés par les expressions discordantes dans les émissions télévisées algériennes Controverse et Place au débat. En effet, sur l'ensemble de ces actes contradictoires, ce sont les désaccords non-polis qui dominent avec un pourcentage de 77,8\%. Moins fréquents que les désaccords non-polis, les désaccords polis mais durcis présentent un pourcentage de $20,7 \%$. Les désaccords poli-rudes sont en revanche très rares dans le corpus couvrant un pourcentage de $1,5 \%$. Par ailleurs, nous ne rencontrons aucune manifestation de l'impolitesse ni de la politesse non renforcée (au contraire, tous les désaccords sont renforcés dans le corpus).

\section{Conclusion}

Dans les émissions Controverse et Place au débat, l'expression du désaccord génère, selon sa formulation, des actes perlocutoires de politesse variés. Nous avons ainsi distingué des désaccords polis mais renforcés, non-polis ainsi que ceux que nous proposons d'appeler des désaccords « poli-rudes ». Cependant, malgré cette diversité, ce sont les désaccords non-polis qui l'emportent dans ces émissions, contrairement aux autres effets (politesse avec renforçateurs et polirudesse) qui se présentent à petites doses. Cela ne sort pas des normes du débat qui consistent selon Kerbrat-Orecchioni $(2010: 41)$ "à éviter aussi bien la politesse excessive que l'impolitesse ostensible ». Par ailleurs, l'effet de polirudesse est produit à petite fréquence dans ces émissions, ce qui pourrait être expliqué par la rareté des expressions ironiques dans ce contexte qui appelle un registre sérieux. De plus, aucun effet de politesse non renforcée n'est observé dans le corpus, ce qui est normal dans ce contexte puisque la raison de la participation des interactants à ces débats est de donner leurs points de vue et de les argumenter et non pas de se jeter des fleurs.

\section{Annexe : Convention de transcription du corpus}

Toutes les productions verbales sont transcrites en minuscules, la majuscule renvoie au segment accentué et les chiffres sont notés en lettres. Les segments correspondant aux phénomènes analysés sont soulignés. L'identifiant est en début du tour et composé de deux caractères. L'énumération se fait à base de changement des tours de parole et non de lignes.

$/$ : intonation montante

$\backslash$ : intonation descendante

$[$ : chevauchement et interruption

(.) : pause 
$=:$ enchainement rapide

$:::$ : allongement

- : troncation

euh : hésitation

$[\ldots]$ : coupure dans un extrait du corpus

\section{Les participants}

KD (Khaled Drareni), l'animateur des émissions Controverse et Place au débat.

AG (Abdelkrim Ghezali), journaliste, ancien directeur de la rédaction de la Tribune.

ML (Mouloud Lounaouci), chercheur, auteur, membre fondateur du Mouvement Culturel Berbère.

OA (Omar Ait Mokhtar), Représentant du Mouvement Citoyen des Algériens de France.

SK (Sidali Kouidri Filali), Représentant du Mouvement Barakat.

AA (Abdelmadjid Attar), ancien Président Directeur Général de Sonatrach, ancien Ministre des ressources en eau.

KB (Khaled Boukhelifa), expert en énergie, ancien Directeur Général au Ministère d'énergie et des mines.

SR (Sabrina Rahmani), médecin et membre de la société civile.

CC (Chems Eddine Chitour), chercheur et spécialiste en énergie, professeur émérite à l'école nationale polytechnique d'Alger

HS (Hakim Soufi), PDG de Macir Vie ${ }^{14}$, membre du directoire de Jil FCE.

MR (Mélissa Roumadi), Journaliste économique, Directrice du magazine Cap Algérie.

SB (Saida Benhabiles), présidente du « Croissant-Rouge-Algérien ».

\section{Références}

Angouri Jo \& Locher Miriam, 2012, « Theorising disagreement », Journal of Pragmatics, $\mathrm{n}^{\circ} 44$, pp. 1549-1553.

Austin John Langshaw, 1970, Quand dire, c'est faire, Paris : Seuil.

Doury Marianne, 2009, " "Sans faire allégeance” : l'euphémisme dans l'expression des accords et désaccords dans un débat politique », Synergies Italie $\left(\mathrm{N}^{\circ}\right.$ spécial « Euphémismes et stratégies d'atténuation du dire »), pp. 111-122.

Doury Marianne \& Kerbrat-Orecchioni Catherine, 2011, «La place de l'accord dans l'argumentation polémique : le cas du débat Sarkozy/Royal (2007) », A contrario, n 16 , pp. 63-87, http://www.cairn.info/revue-a-contrario-2011-2.html.

Goffman Erving, 1973, La mise en scène de la vie quotidienne.1. La présentation de soi. 2. Les relations en public, Paris : Minuit.

Goffman Erving, 1974, Les rites d'interaction, Paris : Minuit.

Hocini Siham \& Bedjaoui Wafa, 2018a, «L'expression du désaccord dans le débat télévisé algérien : cas de l'émission Controverse », Traduction \& Langues, n¹7, pp. 138-156, https://www.asjp.cerist.dz/en/article/59447.

Hocini Siham \& Bedjaoui Wafa, 2018b, «Les modalités de production du désaccord dans l'émission télévisée algérienne Place au débat : approche interactionnelle », Dirassat \& Abhath 33 (études et recherches), pp. 1012-1022, https://www.asjp.cerist.dz/en/PresentationRevue/20.

Kerbrat-Orecchioni Catherine, 1992, Les interactions verbales, tome 2, Paris : Armand Colin.

\footnotetext{
${ }^{14}$ Macir Vie est une compagnie d'assurance privée algérienne spécialisée dans les assurances de personnes dans les domaines du voyage, de la vie et de la santé.
} 
Kerbrat-Orecchioni Catherine, 1994, Les interactions verbales, tome 3, Paris : Armand Colin.

Kerbrat-Orecchioni Catherine, 1995, «La construction de la relation interpersonnelle: quelques remarques sur cette dimension du dialogue ", Cahiers de linguistique française, $\mathrm{n}^{\circ} 16$, pp. 69-88.

Kerbrat-Orecchioni Catherine, 2005, Le discours en interaction, Paris : Armand Colin.

Kerbrat-Orecchioni Catherine, 2010, «L'impolitesse en interaction : Aperçus théoriques et étude de cas », Lexis Special, n², pp. 35-60, En ligne: Consulté le 01 mars 2019], URL : [https://journals.openedition.org/lexis/796.

Kerbrat-Orecchioni Catherine, 2016, «Le désaccord, réaction "non préférée" ? Le cas des débats présidentiels ", Cahiers de praxématique, $\mathrm{n}^{\circ} 67$, pp. 11-30, http://praxematique.revues.org/4524, consulté le 09/10/2017.

Kerbrat-Orecchioni Catherine, 2017, Les débats de l'entre-deux-tours des élections présidentielles françaises. Constantes et évolutions d'un genre, Paris : L'Harmattan.

Moïse Claudine et Oprea Alina, 2015, « Présentation. Politesse et violence verbale détournée », Semen $\mathrm{n}^{\circ} 40$, https://journals.openedition.org/semen/10387, consulté le 8/11/ 2019.

Pomerantz Anita, 1978, "Compliment Responses », in Schenkein J. (éd.), Studies in the Organization of Conversational Interaction, New York : Academic Press, pp. 79-112.

Pomerantz Anita, 1984, «Agreeing and disagreeing with assessments: some features of preferred/dispreferred turn shapes ", in Atkinson J.M. \& Heritage J. (éds.), Structures of Social Action, Cambridge : CUP, pp. 57-101.

Searle John Rogers, 1972, Les actes de langage, Paris : Hermann.

Vion Robert, 2008, La Communication Verbale. Analyse des Interactions, Paris : Hachette Supérieur.

Zongo Bernard, 2004, Le parler ordinaire multilingue à Paris. Ville et alternance codique, Paris : L'Harmattan. 


\section{GLOTTOPOL}

Revue de sociolinguistique en ligne

Comité de rédaction : Michaël Abecassis, Salih Akin, Sophie Babault, Claude Caitucoli, Véronique Castellotti, Régine Delamotte, Robert Fournier, Stéphanie Galligani, Emmanuelle Huver, Normand Labrie, Foued Laroussi, Benoit Leblanc, Fabienne Leconte, Gudrun Ledegen, Danièle Moore, Clara Mortamet, Alioune Ndao, Isabelle Pierozak, Gisèle Prignitz.

Rédactrice en chef : Clara Mortamet.

Comité scientifique : Claudine Bavoux, Michel Beniamino, Jacqueline Billiez, Philippe Blanchet, Pierre Bouchard, Ahmed Boukous, Pierre Dumont, Jean-Michel Eloy, Françoise Gadet, Monica Heller, Caroline Juilliard, Jean-Marie Klinkenberg, Jean Le Du, Marinette Matthey, Jacques Maurais, Marie-Louise Moreau, Robert Nicolaï, Didier de Robillard, Paul Siblot, Claude Truchot, Daniel Véronique.

\section{Comité de lecture pour ce numéro :}

Mickael Abecassis, Michelle Auzanneau, Salih Akin, Céline Amourette, Gabriel Bergounioux, Philippe Boula de Mareuil, Catherine Brissaud, Aude Bretegnier, Maria Candea, Véronique Castellotti, Régine Delamotte, Jean-François De Pietro, Catherine Delarue-Breton, Anne Dister, Alexandre Duchêne, Valentin Feussi, Françoise Gadet, Stéphanie Galligani, François Gaudin, Monica Heller, Emmanuelle Huver, Caroline Juilliard, Abou Bakri Kebe, Jean-Marie Klinkenberg, Christian Lagarde, Foued Laroussi, Fabienne Leconte, Evelyne Lloze, Nolwenn Lorenzi, Gudrun Ledegen, Nadja Maillard, Marinette Matthey, Bruno Maurer, Véronique Miguel Addisu, Saskia Mugnier, Claire Oger, Marielle Rispail, Richard Sabria, Véronique Traverso, Christel Troncy, Cécile Van den Avenne. 